

\title{
Bearing defect classification based on individual trained wavelet kernel local fisher discriminant analysis with particle swarm optimization
}

Van, M., \& Kang, H-J. (2015). Bearing defect classification based on individual trained wavelet kernel local fisher discriminant analysis with particle swarm optimization. IEEE Transactions on Industrial Informatics, 12(1), 124. https://doi.org/10.1109/TII.2015.2500098

Published in:

IEEE Transactions on Industrial Informatics

Document Version:

Peer reviewed version

Queen's University Belfast - Research Portal:

Link to publication record in Queen's University Belfast Research Portal

Publisher rights

Copyright 2015 IEEE. This work is made available online in accordance with the publisher's policies. Please refer to any applicable terms of use of the publisher.

\section{General rights}

Copyright for the publications made accessible via the Queen's University Belfast Research Portal is retained by the author(s) and / or other copyright owners and it is a condition of accessing these publications that users recognise and abide by the legal requirements associated with these rights.

Take down policy

The Research Portal is Queen's institutional repository that provides access to Queen's research output. Every effort has been made to ensure that content in the Research Portal does not infringe any person's rights, or applicable UK laws. If you discover content in the Research Portal that you believe breaches copyright or violates any law, please contact openaccess@qub.ac.uk. 


\title{
Bearing Defect Classification based on Individual Wavelet Local Fisher Discriminant Analysis with Particle Swarm Optimization
}

\author{
Mien Van, Hee-Jun Kang
}

\begin{abstract}
In order to enhance the performance of bearing defect classification, feature extraction and dimensionality reduction have become important. In order to extract the effective features, wavelet kernel local fisher discriminant analysis (WKLFDA) is first proposed; herein, a new wavelet kernel function is proposed to construct the kernel function of LFDA. In order to automatically select the parameters of WKLFDA, a particle swarm optimization (PSO) algorithm is employed, yielding a new PSO-WKLFDA. When compared to the other state-of-the-art methods, the proposed PSO-WKLFDA yields better performance. However, the use of a single global transformation of PSO-WKLFDA for the multiclass task does not provide excellent classification accuracy due to the fact that the projected data still significantly overlap with each other in the projected subspace. In order to enhance the performance of bearing defect classification, a novel method is then proposed by transforming the multiclass task into all possible binary classification tasks using a one-against-one (OAO) strategy. Then, individual PSO-WKLFDA (I-PSO-WKLFDA) is used for extracting effective features of each binary class. The extracted effective features of each binary class are inputted to a support vector machine (SVM) classifier. Finally, a decision fusion mechanism is employed to merge the classification results from each SVM classifier in order to identify the bearing condition. Simulation results using synthetic data and experimental results using different bearing fault types show that the proposed method is well suited and effective for bearing defect classification.
\end{abstract}

Index Terms-Local fisher discriminant analysis, bearing defect classification, wavelet kernel, feature extraction, dimensional reduction, pattern recognition.

\section{INTRODUCTION}

$\mathrm{R}$ OTATING machines are widely used in the manufacturing industry. Bearings play a significant role in modern rotating machinery, and their carrying capacity and reliability are crucial for overall machine performance. Since bearings frequently fail and can consequently lead to catastrophic

Copyright (c) 2011 IEEE. Personal use of this material is permitted. However, permission to use this material for any other purposes must be obtained from the IEEE by sending a request to pubs-permissions@ieee.org.

Mien Van is with the Advanced Robotic Centre, National University of Singapore, Singapore (e-mail: vamien1@gmail.com).

Hee-Jun Kang, was with School of Electrical Engineering, University of Ulsan, South Korea (e-mail: hjkang@ulsan.ac.kr). system failure, it is important to be able to automatically detect and rapidly and accurately diagnose the existence and severity of bearing faults.

During the past decades, signal analysis-based fault diagnosis methods have been widely used to identify multiple bearing defects. This tool extracts fault features and then identifies fault patterns. In this field, two major approaches have been widely developed for bearing fault diagnosis: acoustic signal analysis and vibration signal analysis [1]. Among these, vibration signal-based diagnosis has become the most popular monitoring technique because of its ease of measurement. However, the difficulty of defect detection and classification in bearings from vibration data is that the signature of a defective bearing presents a wide frequency band and can be overwhelmed by noise [2].

In literature, there are two approaches to diagnose the bearing faults. The first approach is based on identifying the bearing characteristic frequency (BCF) of vibration spectra [3]. This approach, however, is not effective when the signal in heavy noise. It means that the absence of clear BCF due to noise should not be informed as a completely healthy condition of the bearing [10]. In the second approach, fault diagnosis can be regarded as a problem concerning pattern recognition, which mainly includes three important stages: feature calculation, feature extraction and dimensionality reduction, and pattern classification [4-5]. In order to acquire more fault information and improve the accuracy of the diagnosis system, many feature calculation methods have been developed based on three domains: time domain analysis, frequency domain analysis, and time-frequency domain analysis [6-7]. Recently, time-frequency analysis based on empirical mode decomposition (EMD) has been developed as an efficient method to extract bearing characteristics [8]. However, EMD is sensitive to measurement noise, which decreases the performance of feature extraction. To increase the performance of EMD for feature extraction, a hybrid nonlocal means (NLM) de-noising was developed in our previous work [9]. Based on three domains, many features can be calculated from a vibration signal to generate a rich feature set, which may contain many aspects to represent the bearing defect condition. In this way, the subjects of bearing defect identification can be transformed into a pattern recognition problem with high-dimensional data. However, dealing with high-dimensional data has always been a major problem in pattern recognition. High-dimensionality data processing 
suffers many difficulties in real applications such as need to massive computational resources and storage capacity. Meanwhile, the constructed features are often correlated, and not all of them are useful for the specific classification task [10]. Therefore, before employing classification tasks, a second step, dimensionality reduction (DR), should be employed to increase the performance of the classification system. There are two approaches for dimensional reduction: feature selection [11-12] and feature transformation [13-14]. Feature selection aims to find the most important features from a given set of features, while feature transformation aim to obtain low-dimensional feature representation with enhanced discrimination power. Conventional dimensionalityreduction techniques based on feature transformation include unsupervised approaches, such as principle component analysis (PCA), and supervised approaches, such as fisher discriminant analysis (FDA) [13]. Since FDA aims to identify projections with the most discriminant information, whereas PCA-based methods identify projections with minimal reconstruction errors, FDA-based methods generally perform better than PCA-based methods [13]. In [14], an orthogonal variant of FDA, which is called trace ratio linear discriminant analysis (TRLDA), has been developed in order to eliminate redundant information from the scatter matrices in LDA. However, the FDA and its variant TRLDA tend to produce undesired results if data samples in a class are multimodal. According to our experiment, measurement noise and feature extraction in different domain are the main sources generated multimodal of bearing vibrations signal. In [15], multimodal approach has been developed for gearbox fault diagnosis using deep learning. The idea of this approach is to classify the time domain, frequency domain, and time-frequency domain as separate modal feature representations, and then use the deep learning method to learn the modal feature representation. However, this approach also tends to produce undesired results if data samples in a class of a modal feature representation are multimodal. Another approach is to use local fisher discriminant analysis (LFDA) [16], which combines FDA and locality preserving projection (LPP). LFDA considers the local structure of the data samples so that the multimodal data can be appropriately embedded. However, LFDA is a linear method, which makes it difficult to use to describe a complex nonlinear system. To overcome this limitation, the kernel trick-based nonlinear LFDA extensions, called kernel LFDA (KLFDA), have attracted a great deal of attention. The basic idea is to nonlinearly map the input data from the input space to a higher dimensional feature space, where the complex distribution is expected to become linearly separable in the feature space, and then perform LFDA in the feature space. Linear, polynomial, and radial basis function (RBF) kernels are generally used in many applications, among which, RBF kernels are most commonly used [17].

Since the wavelet technique emerged as a powerful tool for nonlinear signal approximation [19], it has been employed as a kernel function (called a wavelet kernel) for support vector machine (SVM) classifiers $[18,20]$. The experimental results shown that the wavelet kernel can provide a better approximation than does the use of the RBF kernel when incorporated into the SVM classifier. This is a valuable motivation for us to study the application of a wavelet kernel for LFDA. However, when applied to a wavelet kernel for LFDA, a problem arises in terms of selection of wavelet kernel parameters so that the KLFDA can generate the highest performance. In addition, in practical applications of LFDA and KLFDA, one of the most important issues is selection of the nearest neighbor parameter $k$-th so that LFDA and KLFDA can be best utilized. Particle swarm optimization (PSO) was proposed by Kennedy and Eberhart [21] as a population-based, stochastic optimization technique inspired by the social behavior of bird flocks or fish schools. Compared to other heuristic optimization methods such as genetic algorithm, simulated annealing, etc., one advantage of the PSO technique, in addition to its high computational efficiency and rapid convergence, is its ability to successfully avoid local minima. PSO has been successfully applied to determine the optimal value of many optimization problems. Therefore, PSO can be used to effectively select the $k$-th and wavelet kernel parameters.

In this paper, a wavelet kernel local fisher discriminant analysis (WKLFDA) is proposed to extract the effective features for bearing defect classification. The optimal parameters of WKLFDA are automatically selected by a PSObased-classifier, which constitutes a new PSO-WKLFDA. However, the use of a single global transformation of PSOWKLFDA for the multiclass task does not provide excellent classification accuracy due to the fact that the projected data still significantly overlap with each other in the projected subspace. In order to enhance the performance of bearing defect classification, a novel method is proposed by transforming the multiclass defect classification subject into a multi-binary defect classification subject. For each binary class, an individual PSO-WKLFDA (I-PSO-WKLFDA) is used to extract effective features. In the literature, the most popular strategies to convert the multiclass classification subject to a multi-binary classification subject are the oneagainst-all (OAA) and one-against-one (OAO) methods [22]. In this paper, both strategies are employed and compared to evaluate these performances. After employing PSOWKLFDA for each binary class, the effective features are used as the input to an SVM classifier. The SVM classifier is used in this paper because it is a well-known method that performs very well for binary classification problems and provides higher classification accuracy using fewer feature inputs compared to other classifiers [22]. Finally, a decision fusion mechanism is employed to merge the classification results from each SVM classifier to identify the bearing condition.

The main contributions of this paper are summarized as follows:

1) This paper claims that the conventional frequency domain approach fail to identify the bearing fault when the signal is immersed in heavy noise.

2) This paper shows that the data, which contains the features extracted from the bearing vibration signal, is a type of multimodal data. Thus, the multimodal dimensional reduction would be a good choice to enhance the classification accuracy. 
3) A new multimodal dimensional reduction, namely IPSO-WKLFDA (OAA-PSO-WKLFDA and OAO-PSOWKLFDA), is proposed to enhance the diagnosis performance.

4) Experiments results for measured bearing vibration data show that the proposed I-PSO-WKLFDA outperforms conventional frequency domain approach, feature selection approach, and other state-of-the-art multimodal DR methods.

The rest of this paper is organized as follows. Section II describes the proposed WKLFD and PSO-WKLFDA methods. The OAA and OAO strategies and the proposed IPSO-WKLFDA method are described in Section III. Section IV presents the construction of a feature set based on time, frequency, and time-frequency domains and the proposed bearing defect identification. Section $V$ provides experimental data to verify the effectiveness of the proposed WKLFDA, PSO-WKLFDA, and I-PSO-WKLFDA algorithms. Finally, concluding remarks are given in Section VI.

\section{WAVELET KERNEL LOCAL FISHER DISCRIMINANT ANALYSIS}

\section{A. Local Fisher Discriminant Analysis (LFDA)}

LFDA [16] is a recent extension of FDA that can effectively handle the multimodal problem. By combining the properties of FDA and an unsupervised manifold technique, i.e., Locality Preserving Projection (LPP), LFDA has the ability to simultaneously preserve both between-class separation and within-class local structure.

Consider a data set with a training sample with $p$ features $\left\{x_{i}\right\}_{i=1}^{n}, x_{i} \in \mathfrak{R}^{p}$ and class labels $\left\{y_{i}\right\}_{i=1}^{n}, \quad y_{i} \in\{1,2, \ldots, c\}$, where $c$ is the number of classes, and $n$ is the total number of training samples. Let $n_{l}$ be the number of training samples available for the $l$ th class, and $\sum_{l=1}^{c} n_{l}=n$. We define $A_{i, j} \in[0,1]$ as the "affinity" between $x_{i}$ and $x_{j}$ given by

$A_{i, j}=\exp \left(-\frac{\left\|x_{i}-x_{j}\right\|^{2}}{\gamma_{i} \gamma_{j}}\right)$

where $\gamma_{i}$ is the local scaling around $x_{i}$, defined by $\gamma_{i}=\left\|x_{i}-x_{i}^{k}\right\|$, and $x_{i}^{k}$ is the $k$-th nearest neighbor of $x_{i}$. $A_{i, j}$ is large if $x_{i}$ and $x_{j}$ are close to each other in the feature space, otherwise it is small. The parameter $k$-th is a tuning factor and is a function of the embedding space. A heuristic choice of $k=7$ has been shown to be effective [23]. However, this value is not applicable for general embedding spaces. In this paper, we use PSO to effectively select this parameter, as described later. In LFDA, the local betweenclass $S^{(l b)}$ and within-class $S^{(l w)}$ scatter matrices are respectively defined as

$S^{l b}=\frac{1}{2} \sum_{i, j=1}^{n} W_{i, j}^{l b}\left(x_{i}-x_{j}\right)\left(x_{i}-x_{j}\right)^{T}$
$S^{l w}=\frac{1}{2} \sum_{i, j=1}^{n} W_{i, j}^{l w}\left(x_{i}-x_{j}\right)\left(x_{i}-x_{j}\right)^{T}$

where $w_{i, j}^{l b}$ and $w_{i, j}^{l w}$ are $n \times n$ matrices respectively defined

as

$$
\begin{aligned}
& W_{i, j}^{l b}= \begin{cases}A_{i, j}\left(1 / n-1 / n_{l}\right), & \text { if } y_{i}=y_{j}=l \\
1 / n & \text { if } y_{i} \neq y_{j}\end{cases} \\
& W_{i, j}^{l w}= \begin{cases}A_{i, j} / n_{l}, & \text { if } y_{i}=y_{j}=l \\
0 & \text { if } y_{i} \neq y_{j}\end{cases}
\end{aligned}
$$

The transformation matrix $W_{L F D A}$ can then be computed by maximizing the local Fisher's ratio $\left(W^{T} S^{l w_{W}}\right)^{-1} W^{T} S^{l b}$ as

$$
W_{\text {LFDA }}=\underset{W}{\operatorname{agrmax}} \frac{\left|W^{T} S^{l b} W\right|}{\left|W^{T} S^{l w} W\right|}
$$

The above optimization problem can be equivalently solved by the generalized eigenvalue decomposition $s^{l b} V=\lambda s^{l w_{V}}$, where $\lambda$ is the generalized eigenvalue, and $V$ denotes the eigenvector that corresponds to a Fisher discriminant direction. Assuming that the generalized eigenvalues $\lambda_{1} \geq \lambda_{2} \ldots \geq \lambda_{p}$ are arranged in descending order, the corresponding generalized eigenvectors $V_{1} \geq V_{2} \ldots \geq V_{p}$ are the localized Fisher discriminant directions of decreasing class severability.

\section{B. Kernel Local Fisher Discriminant Analysis (KLFDA)}

Since LFDA is a linear technique of dimensionality reduction and feature extraction, it tends to provide inaccurate results for complex nonlinear systems. In order to overcome this limitation, KLFDA [17, 18] is presented in this section. KLFDA has been shown to be a very effective feature reduction algorithm in Reproducing Kernel Hilbert Space (RKHS). The kernel trick for LFDA can be explained as follows.

Let $S^{\operatorname{lm}}$ be the local mixture scatter matrix defined by $S^{l m} \equiv S^{l b}+S^{l w}$. From (2)-(5), $S^{l m}$ can be expressed as

$S^{l m}=\frac{1}{2} \sum_{i, j=1}^{n} W_{i, j}^{l m}\left(x_{i}-x_{j}\right)\left(x_{i}-x_{j}\right)^{T}$

where $w_{i, j}^{l m}$ is the $n$-dimensional matrix with the $(i, j)$-th element being

$W_{i, j}^{l m}= \begin{cases}A_{i, j} / n, & \text { if } y_{i}=y_{j}=l \\ 1 / n & \text { if } y_{i} \neq y_{j}\end{cases}$

From (7), $s^{\operatorname{lm}}$ can be expressed as

$$
\begin{aligned}
S^{l m} & =\frac{1}{2} \sum_{i, j=1}^{n} W_{i, j}^{l m}\left(x_{i} x_{i}^{T}+x_{j} x_{j}^{T}-x_{i} x_{j}^{T}-x_{j} x_{i}^{T}\right) \\
& =\sum_{i=1}^{n}\left(\sum_{j=1}^{n} W_{i, j}^{l m}\right) x_{i} x_{i}^{T}-\sum_{i, j=1}^{n} W_{i, j}^{I m} x_{i} x_{j}^{T}
\end{aligned}
$$

which can be expressed in matrix form as $S^{l m}=X L^{l m} X^{T}$ 
where $L^{I m}=D^{l m}-W^{l m}$, and $D^{l m}$ is the $n$-dimensional matrix with the $i$-th diagonal element of $D_{i, i}^{l m}=\sum_{j=1}^{n} w_{i, j}^{l m}$. Similarly, $s^{l b}$ can be expressed in the form $S^{l b}=X L^{l b} X^{T}$, where $L^{l b}=D^{l b}-W^{l b}$, and $D^{l b}$ is an $n$-dimensional matrix with the $i$ th diagonal element of $D_{i, i}^{l b}=\sum_{j=1}^{N} W_{i, j}^{l b}$. Thus, the eigenvector problem $S^{l b} V=\lambda S^{l w_{V}}$ can be expressed as $X L^{l b} X^{T} V=\lambda X L^{l w} X^{T} V$

where $L^{l b}=L^{l m}-L^{l w}$. Since $X^{T} V$ is in the range of $X^{T}$, it can be expressed using some vector $\alpha \in \Re^{n}$ as

$X^{T} V=X X^{T} \alpha=K \alpha$

where $K$ is the $n$-dimensional matrix with the $(i, j)$-th element $K_{i, j} \equiv x_{i}^{T} x_{j}$

Substituting this into (11) and multiplying the resulting equation by $X^{T}$, we obtain

$K L^{l b} K \alpha=\lambda K L^{l w} K \alpha$

This implies that $\left\{x_{i}\right\}_{i=1}^{n}$ appear only in terms of their inner products. Thus, we obtain a nonlinear variant of LFDA using the kernel trick.

We further define a nonlinear mapping $\Phi(\cdot)$ from input space $\mathfrak{R}^{p}$ to a higher-dimensional RKHS $\Omega$ as follows $\Phi: \Re^{p} \rightarrow \Omega, x \rightarrow \Phi(x)$

Then, a kernel function $K$ is defined as $K\left(x_{i}, x_{j}\right)=\left\langle\Phi\left(x_{i}\right), \Phi\left(x_{j}\right)\right\rangle$

where $\langle\cdot, \cdot\rangle$ is the inner product of two vectors.

Commonly used kernel functions include linear, polynomial, and RBF kernels. Among these, the RBF kernel is the most popular and is defined as

$$
K\left(x_{i}, x_{j}\right)=\exp \left(-\frac{\left\|x_{i}-x_{j}\right\|^{2}}{2 \sigma^{2}}\right)
$$

where $\sigma$ is the dilation parameter, which is determined by users.

\section{Wavelet Kernel Local Fisher Discriminant Analysis (WKLFDA)}

The principle of wavelet analysis is to express or approximate a signal or function with a family of functions generated by dilations and translations of a mother wavelet function

$$
h_{a, c}(x)=|a|^{-1 / 2} h\left(\frac{x-c}{a}\right)
$$

where $x, a, c \in \mathfrak{R}, a$ is a dilation factor, $c$ is a translation factor, and $h(x)$ is the mother wavelet, which satisfies the following condition [19]

$$
W_{h}=\int_{0}^{\infty} \frac{|F(\omega)|^{2}}{|\omega|}<\infty
$$

where $F(\omega)$ is the Fourier transform of $h(z)$. The wavelet transform of a function, $g(z)$, can be expressed as

$W_{a, c}(g)=\left\langle g(x), h_{a, c}(x)\right\rangle$

On the right-hand side of (18), $\langle\cdot \cdot\rangle$ denotes the dot product.

Equation (18) represents the decomposition of a function, $g(x)$, on a wavelet basis, $h_{a, c}(x)$.

A wavelet function can be written in the following form:

$h(x)=\sum_{i=1}^{N} h\left(x_{i}\right)$

where $x=\left[x_{1}, x_{2}, \ldots, x_{N}\right]^{T} \in \mathfrak{R}^{N}$. Then, if $x, x^{\prime} \in \mathfrak{R}^{N}$, the dotproduct wavelet kernels can be expressed as

$K\left(x, x^{\prime}\right)=\sum_{i=1}^{N} h\left(\frac{x_{i}-c_{i}}{a}\right) \sum_{i=1}^{N} h\left(\frac{x_{i}^{\prime}-c_{i}^{\prime}}{a}\right)$

and the translation-invariant wavelet kernels are expressed as

$K\left(x, x^{\prime}\right)=\prod_{i=1}^{N} h\left(\frac{x_{i}-x^{\prime}}{a}\right)$

Equation (21) represents a multidimensional wavelet function.

Based on the wavelet function in [19], without loss of generality, we propose a Morlet wavelet function as the translation invariant wavelet kernel function

$h(x)=\cos (\lambda x) \cdot \exp \left(-\frac{x^{2}}{2}\right)$

Considering the mother wavelet defined in (22) and the dilation $a$, the wavelet kernel of this mother wavelet is

$$
\begin{aligned}
K\left(x, x^{\prime}\right) & =\prod_{i=1}^{N} h\left(\frac{x_{i}-x^{\prime}}{a}\right) \\
& =\prod_{i=1}^{N}\left(\cos \left(\lambda \times \frac{\left(x_{i}-x_{i}^{\prime}\right)}{a}\right) \exp \left(-\frac{\left\|x_{i}-x_{i}^{\prime}\right\|^{2}}{2 a^{2}}\right)\right)
\end{aligned}
$$

where $a$ is a parameter of the RBF kernel, and $\lambda$ is a new parameter that controls the kernel shape and must be suitably selected. It can be seen that when $\lambda=0, K\left(x, x^{\prime}\right)$ represents a RBF kernel. When $\lambda=1.5, K\left(x, x^{\prime}\right)$ approximates the Mexhat kernel in the range of $[-1,1]$.

\section{D.Particle Swarm Optimization (PSO) for Parameter Selection of WKLFDA (PSO-WKLFDA)}

The PSO algorithm was first developed by Eberhart and Kennedy in 1995. It is a powerful tool for dealing with global optimization problems [21]. PSO possesses several advantages compared with other heuristic optimization techniques such as simplicity, ease of implementation, robustness to control parameters, and computational efficiency.

In PSO, the population is referred to as a swarm, and the individuals are classed as particles. The ith particle is characterized by its current position vector, $x_{i}(t)=\left(x_{i 1}(t), x_{i 2}(t), \ldots, x_{i D}(t)\right)$, in the search space, where $D$ is the dimensionality of the search space, and the velocity vector is $v_{i}(t)=\left(v_{i 1}(t), v_{i 2}(t), \ldots, v_{i D}(t)\right)$. Each particle maintains a record of its personal best position, $p_{\text {best }, i}(t)=\left(p_{\text {best }, i 1}(t), p_{\text {best }, \text { i2 }}(t), \ldots, p_{\text {best }, i D}(t)\right)$, and the whole swarm of particles maintains a record of the global best 
position, $G_{\text {best }}(t)=\left(g_{\text {best }, 1}(t), g_{\text {best }, 2}(t), \ldots, g_{\text {best }, D}(t)\right)$. Particles move in the search space in order to search for the optimal solution. During the movement, each particle updates its position and velocity according to the distance to its personal best position and the distance to the global best position with the following equations:

$$
\begin{aligned}
& \omega_{k}=\omega_{\max }-\frac{\omega_{\max }-\omega_{\min }}{i t e r_{\max }} \times \text { iter } \\
& v_{i d}^{t+1}=\omega \cdot v_{i d}^{t}+c_{1} r_{1} \cdot\left(p_{\text {best }, i d}^{t}-x_{i d}^{t}\right)+c_{2} \cdot r_{2} \cdot\left(g_{\text {best }, d}^{t}-x_{i d}^{t}\right) \\
& x_{i d}^{t+1}=x_{i d}^{t}+v_{i d}^{t+1}
\end{aligned}
$$

where $\omega_{\min }$ is the minimal inertia weight, and $\omega_{\max }$ is the maximal inertia weight. iter is the current iteration number, iter $_{\max }$ is the maximum iteration number. $t$ represents the $t$ th iteration in the evolutionary process, while $d \in D$ represents the $d$ th dimension in the search space. $c_{1}$ and $c_{2}$ are acceleration constants, and $r_{1}$ and $r_{2}$ are random values uniformly distributed in $[0,1]$.

The WKLFDA feature extraction constructed using the wavelet kernel function defined in (23) has three determined parameters, a nearest neighbor parameter $k(-$ th) and two wavelet kernel parameters $\lambda$ and $a$. In this study, we employ a PSO-based SVM classifier; herein, the classification accuracy of an SVM classifier is used as a fitness function to automatically select the parameters of the WKLFDA feature extraction. The step-by-step implementation details are described below:

Step 1: Initialization The upper and lower limits of the position and the velocity of the particles and parameters such as $c_{1}, c_{2}$, iter $\max , \omega_{\max }, \omega_{\min }$ are initialized. Further, particle swarm with population $N$ is initialized, as is the position and velocity of each particle ( $k, \lambda$ and $a$-parameters for WKLFDA).

Step 2: Evaluation of initial population All the particles are evaluated for fitness based on a cost function. In this step, the following work is performed to obtain the fitness function. The training and validation data are projected onto the modeling WKLFDA subspace to obtain training and validation features. Then, the training and validation features are input into the SVM classifier to obtain classification accuracy. The classification accuracy is used as the fitness function and is defined as follows

$$
\text { fitness function }=\frac{N_{t}}{N_{t}+N_{f}}
$$

where $N_{t}$ and $N_{f}$ denote the number of true and false classifications, respectively. A particle with the high classification accuracy value produces a high fitness value. The $p_{\text {best }}$ of the individual particles and $g_{\text {best }}$ of the population are identified.

Step 3: $\quad$ Updating For each particle, update particle velocity according to Eq. (25) and particle position according to Eq. (26) in order to generate a new swarm.
Step 4: Evaluation of updated population Evaluate the fitness value of the newly updated particles in a process similar to Step 2, and then update the $p_{\text {best }, i}$ and $G_{\text {best }}$ of the swarm. For an individual particle, if the

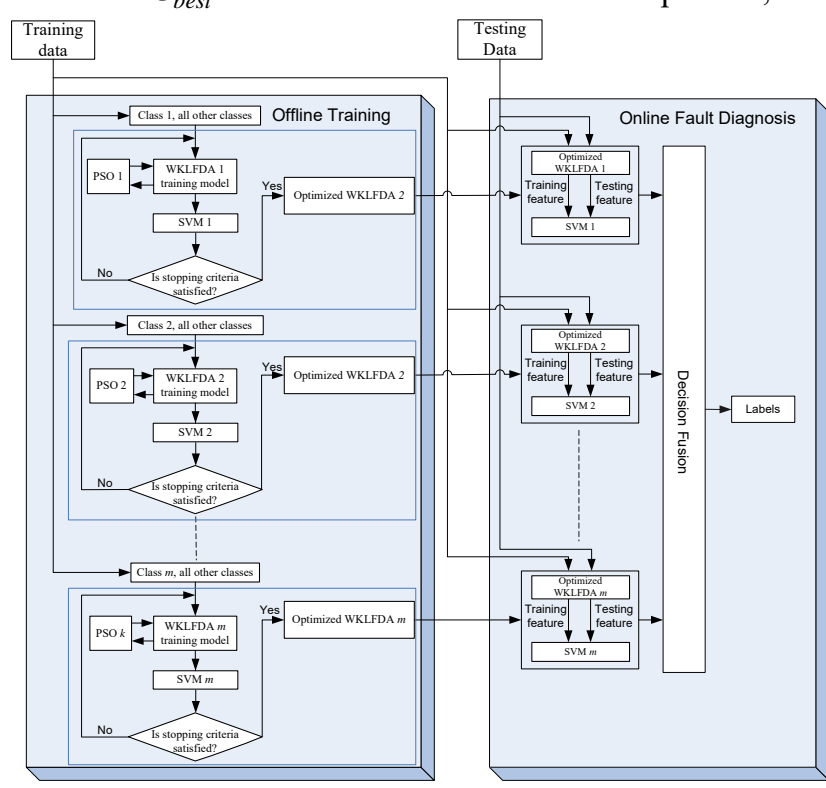

Fig. 1 Structure diagram of individual PSO-WKLFDA.

newly updated fitness value is greater than the historical local best value, the local best position, $p_{\text {best }, i}$, will be replaced by the current position. For the swarm of particles, if the current fitness value is greater than the global best, the global best position, $G_{b e s t}$, will be replaced by the current position.

Step 5: If the maximum number of iterations is not yet reached, return to Step 3. Otherwise, go to Step 6.

Step 6: $\quad$ Select the global best position, $G_{\text {best }}$, in the swarm as the ultimate solution. The value encoded from the global best position, $G_{\text {best }}$, is assigned as the optimal value for the parameters of the WKLFDA algorithm.

In order to increase the robustness, a $k$-fold CV method [24] is employed to compute the fitness function in Step 2. In the $k$ -

fold $\mathrm{CV}$ estimation procedure, the training data is randomly divided into $k$ equal size subsamples. Of the $k$ subsamples, the $k-1$ subsamples are used as the training data, and the remaining subsample is retained as the validation data for testing the model. We repeat this procedure until each of the subsamples

is used as a validation set. The $k$ results are then averaged to produce a single estimation. The averaged classification accuracy is used to gauge the fitness of the PSO algorithm. In this study, $k$ is set to 5 . This is a reasonable compromise considering the computational complexity and modeling robustness. 


\section{INDIVIDUAL PSO-WKLFDA}

\section{A. One-Against-All (OAA) Strategy}

The OAA strategy [22] has been applied widely for SVM classification, in which an $m$ class classification problem is divided into $m$ binary class classification problems. Each problem involves a binary classifier, which is responsible for distinguishing one of the classes from all other classes combined. Using a Winner-Takes-All strategy, each binary classifier is trained considering the samples from one of the classes as positive and the samples from all other classes as negative. The decision of the multiclassifier is chosen from the class whose binary classifier provides the greatest output. Formally, given a vector $y=f(x)$ with the outputs of the binary classifiers, the multiclassifier generates a vector $L=\left(l_{1}, \ldots, l_{m}\right)$ in the following way

$i^{*}=\underset{i=1, \ldots, m}{\operatorname{agr} \max } f_{i}(x)$

$L_{i}= \begin{cases}+1 & \text { if } i=i^{*}, i=1, \ldots, m \\ -1 & \text { otherwise }\end{cases}$

\section{B. One-Against-One (OAO) Strategy}

The OAO strategy divides an $m$ class classification problem into $m(m-1) / 2$ binary class classification problems. Each problem involves a binary classifier, which is responsible for distinguishing the samples of one class from the samples of another class. Each binary classifier is trained considering the samples from one of the classes as positive and the other class as negative. Formally, each classifier considers two information classes $l$ and $s \quad(l, s \in\{1,2, \ldots, C\}, l \neq s)$ via a decision function $g_{l, s}(x)$. The global decision function is

$$
N_{l}(x)=\sum_{\substack{s=1 \\ s \neq l}}^{C} \operatorname{sgn}\left(g_{l, s}(x)\right)
$$

where $N_{l}(x)$ is the number of times that class $l$ is assigned for the testing vector $x$. The final class label is assigned according to

$y^{*}=\arg \max _{i=1, \ldots, m} N_{l}(x)$

\section{C.Individual PSO-WKLFDA}

Although the proposed WKLFDA and PSO-WKLFDA algorithms yield better performance than previous state-ofthe-

art approaches, the use of a single global transformation for the multiclass task does not provide excellent classification accuracy due to the fact that the projected data still overlap with each other in the projected subspace. In this paper, we consider the OAA and OAO strategies to reduce the overlapped data in the projected subspace. In the following, we present the proposed method based on OAA frame work (the method based on OAO frame work can be executed as a similar way). Fig. 1 illustrates the proposed classification framework based on OAA strategy. In this way, the $m$ class classification subject is first transformed into an $m$ binary class classification subject. For each binary class, an individual PSO-WKLFDA is used to extract the effective feature. In contrast to the conventional approach, which uses FDA, LFDA, or WKLFDA for the $m$ class classification problem to obtain a single global transformation, this paper use $m$ individual PSO-WKLFDAs for the $m$ binary class classification problem in order to obtain $m$ single transformations. The parameters of each WKLFDA are individually selected by PSO so that the corresponding binary classifier yields the best classification accuracy. The effective features extracted by each WKLFDA are used as the input to

TABLE I

PARAMETERS OF TIME-DOMAIN FEATURES.

\begin{tabular}{|c|c|}
\hline Feature & Equation \\
\hline Standard deviation & $x_{\text {std }}=\sqrt{\frac{\sum_{n=1}^{N}\left(x(n)-x_{m}\right)^{2}}{N}}$ \\
\hline Peak & \multirow{2}{*}{$\begin{array}{l}x_{P}=\max |x(n)| \\
x_{S k e}=\frac{\sum_{n=1}^{N}\left(x(n)-x_{m}\right)^{3}}{(N-1) x_{\text {std }}^{3}}\end{array}$} \\
\hline Skewness & \\
\hline Kurtosis & $x_{k u r}=\frac{\sum_{n=1}^{N}\left(x(n)-x_{m}\right)^{4}}{(N-1) x_{s t d}^{4}}$ \\
\hline Crest factor & $C F=\frac{x_{P}}{x_{r m s}}$ \\
\hline Root mean square & $x_{r m s}=\sqrt{\frac{\sum_{n=1}^{N}(x(n))^{2}}{N}}$ \\
\hline Clearance factor & $C L F=\frac{x_{P}}{\left(\frac{1}{N} \sum_{n=1}^{N} \sqrt{|x(n)|}\right)^{2}}$ \\
\hline Shape factor & $S F=\frac{x_{r m s}}{\frac{1}{N} \sum_{n=1}^{N}|x(n)|}$ \\
\hline Impulse factor & \multirow{2}{*}{$\begin{array}{l}I F=\frac{x_{P}}{\frac{1}{N} \sum_{n=1}^{N}|x(n)|} \\
\text { series for } n=1,2, \ldots, N \text {, and } N \text { is the }\end{array}$} \\
\hline $\begin{array}{l}\text { where } x(n) \text { is a sig } \\
\text { number of data poin }\end{array}$ & \\
\hline
\end{tabular}

the classifier. In this paper, the SVM classifier is used. Finally, a decision-fusion mechanism is employed to merge the classification results from each classifier based on the OAA strategy.

\section{PROPOSED DIAGNOSIS METHODOLOGY}

\section{A. Feature Calculation}

In order to measure the changes in vibration signal due to diverse bearing defects, a vibration sensor is generally attached to a non-rotating part of the machinery, (e.g., bearing housing). Although the bearing housing is the closest element on which to place a vibration sensor, the distance from the source of bearing failures causes the vibration signal to be overwhelmed by noise due to the effects of other components. Thus, the vibration signal should be effectively de-noised 
before being used for analysis. In this paper, we employed the hybrid NLM and EMD method, which had been effectively developed in our previous work [9], to extract the effective features. In this method, the raw vibration signal is first preprocessed using the NLM algorithm to eliminate or reduce the measurement noise; the de-noised signal is then decomposed using EMD to obtain a number of reliable intrinsic mode functions (IMFs). Based on the EMD algorithm, the de-noised signal, $x(t)$, can be decomposed into a number of IMFs, $C(t)$ :

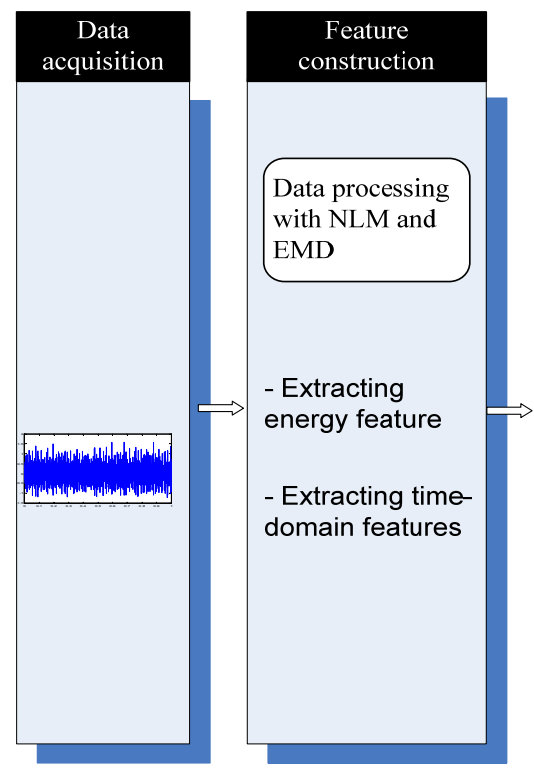

Fig. 2. Structure diagram of bearing defect classification.

$x(t)=\sum_{j=1}^{n} C_{j}(t)+r_{n}(t)$

The first IMF, $C_{1}(t)$, contains mostly high-frequency components. The IMFs $C_{2}(t), C_{3}(t), \ldots, C_{n}(t)$ include different frequency bands ranging from high to low, and $r_{n}(t)$ usually does not contain any signal information. Since the diverse bearing failures exists primarily in mid-and high frequency bands [12], we choose the first four IMFs as the most components for calculating fault signature because they represent the mid- and high-frequency components.

When a rolling bearing with different faults is used in the operation, different resonance frequency components are produced in the vibration signals. The energy of the fault vibration signal depends on the frequency band. To illustrate this change, IMF energy features are introduced in this paper.

For the first four IMF components, the total signal energy of each IMF component can be calculated. The energy of $C_{j}(t)$ is expressed as $E_{j}(t)$, so

$E_{j}=\int\left|C_{j}(t)\right|^{2} d t$

To encompass the large value of $E_{j}(t)$, the normalized energy of the IMF is defined as

$T_{j}=E_{j} / T$

where $T$ is defined as
$T=\left(\sum_{j=1}^{4}\left|E_{j}\right|^{2}\right)^{1 / 2}$

When faults occur in rotating machinery, the vibration signals may change. The amplitude and distribution of the time-domain signals may be different from those of normal bearings. In this paper, the nine time-domain dimensionless parameters including standard deviation, peak, skewness, kurtosis, crest factor, root mean square, clearance factor, shape factor and impulsive factor, described in Table I are extracted
Feature extraction \& Fault identification

from the de-noised signal and the first four IMFs. These features represent the energy, vibration amplitude and time series distribution of the signal in the time domain. Finally, a feature set containing 49 features $(4+9+9 \times 4=49$, i.e., four energy features, nine time-domain features extracted from the de-noised signal, and $9 \times 4=36$ time-domain features extracted from the first four IMFs) is generated to represent a bearing condition.

\section{B. Support Vector Machine (SVM) Classifier}

The SVM binary classifier locates a hyperplane between the two categories with the largest margin in the feature space. This hyperplane is used to classify test samples into one of the two categories.

Given a training set of instances and class label pairs $\left(x_{i}, y_{i}\right), \quad i=1 \ldots l, \quad$ where $\quad x_{i} \in \mathfrak{R}^{n}$ and $y_{i} \in\{1,-1\}^{l}$, the following minimization optimal problem can be solved to find the optimal hyperplane to separate the two categories:

$$
\min _{w, b, \xi} \frac{1}{2}|w|^{2}+C\left(\sum_{i=1}^{l} \xi_{i}\right)
$$

subject to $y_{i}\left(w^{T} \phi\left(x_{i}\right)+b\right) \geq 1-\xi_{i},(\forall i) \xi_{i} \geq 0$

where $w$ is a normal vector to the hyperplane, $b$ is a constant such that $b /\|w\|$ represents the Euclidean distance between the 
hyperplane and the original of the feature space, $\phi$ is a nonlinear function to map the original feature space into the high-dimensional nonlinear feature space. Parameter $C>0$ is the penalty factor of the error term and may be seen as a factor that controls the tradeoff between separation margin and training errors, and $\xi_{i}$ are slack variables that measure the degree of misclassification.

The minimization optimal problem defined in (35) can be written in dual form by applying Lagrange optimization as follows:

$$
\begin{aligned}
\max & \left\{\sum_{i} \alpha_{i=1}^{l} \alpha_{i}-\frac{1}{2} \sum_{i, j=1}^{l} \alpha_{i} \alpha_{j} y_{i} y_{j} \phi\left(x_{i}\right)^{T} \phi\left(x_{j}\right)\right\} \\
\text { subject to } & \sum_{i=1}^{l} \alpha_{i} y_{i}=0,0 \leq \alpha_{i} \leq C, \forall i=1,2, \ldots, l,
\end{aligned}
$$

where the $\alpha_{j}^{\prime} s$ are Lagrange multipliers, and $x_{i}$ and $x_{j}$ are any two different samples in the training dataset. Furthermore, $K\left(x_{i}, x_{j}\right)=\phi\left(x_{i}\right)^{T} \phi\left(x_{j}\right)$ represents the kernel function. Kernels, which are often selected based on the data structure and type of the boundaries between classes, can take many forms such as linear, polynomial, radial basis function, and sigmoid. In this paper, the RBF kernel is used:

$$
K\left(x_{i}, x_{j}\right)=e^{-\gamma\left\|x_{i}-x_{j}\right\|}
$$

where $\gamma$ is an adjustable parameter to be carefully tuned.

\section{Bearing Defect Classification based on Individual PSO- WKLFDA and SVM Classifier}

Based on the above presented feature extraction method and proposed individual PSO-WKLFDA approach, a new bearing fault diagnosis approach is proposed to classify the data mixed with both normal and multiple types of faulty data. The bearing defect classification is based on NLM and EMDbased feature calculation, individual PSO-WKLFDA-based feature extraction and dimensionality reduction, and the SVM classifier. The proposed procedure is described in Fig. 2 and includes the following steps.

Step 1: Vibration signals acquired from the machine are preprocessed using NLM algorithm and EMD in order to obtain IMFs effectively.

Step 2: $\quad$ Extract the energy- and time-domain dimensionless feature parameters from the de-noised signal and the first four IMFs in order to obtain a combined feature set.

Step 3: Building data set and partition into a training data set and testing data set.

Step 4: Convert multiclass classification problem into a number of binary class classification problem based on OAA (or OAO) strategy.

Step 5: Offline Training: this step is done to obtain the optimal PSO-WKLFDA model for each binary class. The training data is used at this step. For each binary class, select the wavelet kernel function for WKLFDA feature extraction as in (23) and then select its parameters using the PSO- based SVM classifier in order to establish an optimal
WKLFDA model for each binary class. After this step, we will obtain an $m$ optimized PSOWKLFDA model for corresponding $m$ binary class, and then use it for the online fault diagnosis.

Step 6: Online Fault Diagnosis:

i) For each binary class, project the training and testing data sets onto the optimized WKLFDA to obtain testing features and training features for each binary class.

ii) The training and testing features are inputted into the SVM classifiers.

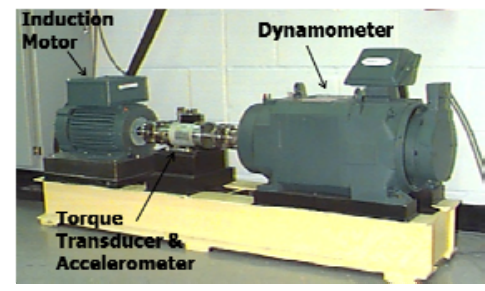

Fig. 3. Experimental setup for vibration monitoring in the Case Western Reserve University Bearing Data [25].
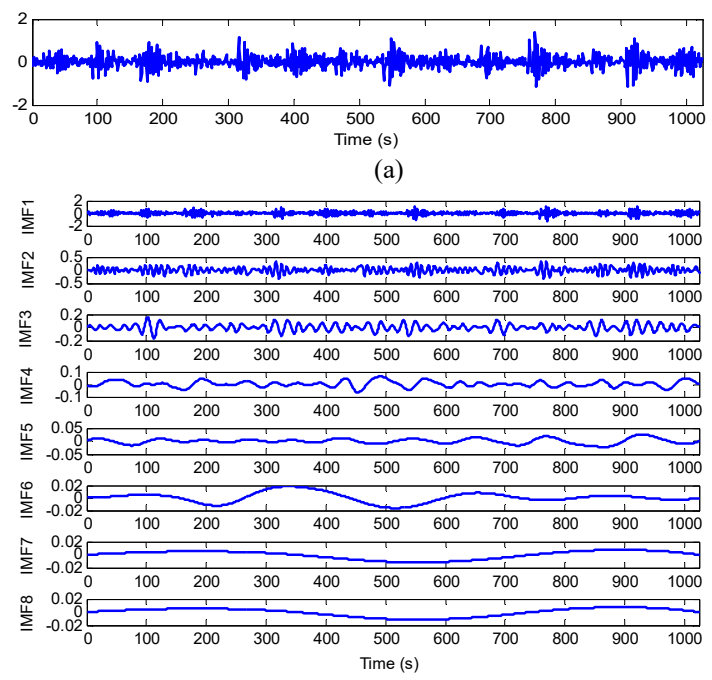

(b)

Fig. 4. (a) De-noised signal of a window after NLM de-noising, and b) its IMF components after EMD decomposition.

iii) Finally, a decision fusion mechanism is employed to merge the classification results from each SVM classifier to identify the bearing condition (labels).

\section{V.EXPERIMENTAL RESULTS}

\section{A. Training and Test Data Configuration}

To validate the proposed method for bearing defect recognition, measured bearing vibration data is used as an example. The vibration data used for analysis are taken from the Case Western Reserve University Bearing Data Center (2014) [25]. The test stand, shown in Fig. 3, consists of a $2 \mathrm{hp}$ motor (left), a torque transducer/encoder (center), a dynamometer (right), and control electronics (not show). Test bearings support the motor shaft. Single-point faults with diameters of 7, 14, and 21 mil were introduced in the test 
bearings using electro-discharge machining (1 mil $=0.001$ inches). Vibration data is collected using accelerometers, which are attached to the housing with magnetic bases. Accelerometers were placed at the three o'clock position on the drive end of the motor housing. Vibration signals were collected using a 16-channel operating conditions: $\{1\}$ normal condition, $\{2\}$ outer race fault (ORF), $\{3\}$ inner race fault (IRF), and $\{4\}$ ball fault (BF). Each fault condition includes three different sizes, 0.007, 0.014, and $0.021 \mathrm{in}$. Hence, there are ten conditions (10 classes) that need to be identified in this experiment. All the experiments were done for one load condition ( $3 \mathrm{hp}$ ), where the rotation speed was $1730 \mathrm{r} / \mathrm{min}$. The sampling rate was $12,000 \mathrm{~Hz}$. The collected raw vibration signals were divided into sections of equal window lengths. Each window contains 1024 point. For each window, NLM and EMD are employed to obtain IMF components. Fig. 4a) shows the time domain of a window after NLM de-noising, and Fig. 4b) shows the de-noised signal IMF components after EMD decomposition. From this figure, we can see that the first four IMF components represent the mid- and high frequency components of the original signal. Hence, we used the first four IMFs for analysis as discussed above. Then, a set of features (49 features) as defined in Section III was then constructed from each denoised signal and the first four IMFs of each window were used to represent the characteristic of the vibration signals. For each condition, 100 samples were used, and therefore the whole dataset corresponding to the ten signal conditions consists of 1000 samples.

\section{B. Performance Evaluation}

In order verify the four main contributions of this paper, as described in the introduction part, the experiment set is divided into four subsets: 1) the performance of the conventional frequency domain analysis is analyzed, 2) verifies that the bearing fault data is a type of multimodal data, 3) the performance of the proposed method is compared with other state-of-the-art multimodal dimensional reduction methods, and 4) we compare the performance of the proposed method with the used of lower dimensional data and feature selection methods.

Firstly, we analyze the performance of the conventional frequency domain analysis to see its performance. According to [9], after NLM and EMD decomposition, the first IMF is passed through the envelope to identify the BCFs, which can be used to detect and isolate the fault. First, we consider the inner race faults that are $0.007,0.014$, and 0.021 inches thick. The results of the FFT plots of the first IMF are shown in Fig. $5 a), b), c)$, respectively. From these figures, we can see that the peaks corresponding to the inner race fault frequency can be easily identified in Fig. 5a) and Fig. 5c), but fail to identify in Fig. 5b). Similar analysis is executed for outer race faults of $0.007,0.014$, and 0.021 inches thick. The FFT plots are shown in Fig. 5d), e) and f), respectively. From the results, we can see that the characteristic frequency of the outer race fault and its harmonic are obvious in Figs. 5d) and 5f), but unseen in Fig. 5e). From the results, we can conclude that the conventional frequency domain analysis is sometime fail to extract BCFs for the noisy vibration signal.

Secondly, the distribution of the samples of the bearing data is analyzed. Fig. 6 shows the sample distribution of three example classes. It is obvious to see that the data, which contains the extracted features of the bearing fault signal, is a type of multimodal data. Thus, multimodal dimensional reduction technique would be a good choice to enhance the performance of the bearing fault diagnosis.

Thirdly, we compare the performance of the proposed methods with other state-of-the-art multimodal dimensional reduction methods. In this paper, we developed three

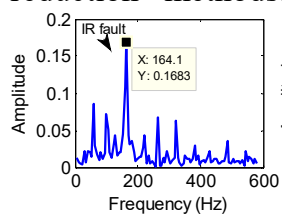

(a)

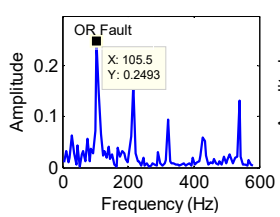

(d)

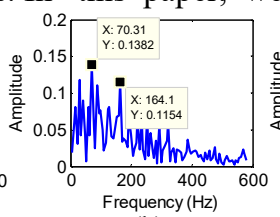

(b)

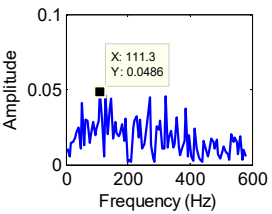

(e)

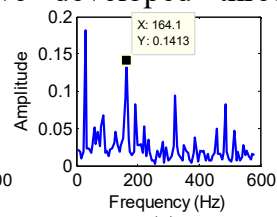

(c)

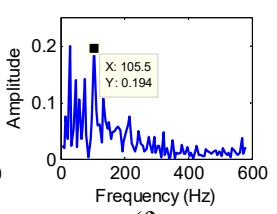

(f)
Fig. 5. Frequency domain analysis based on hybrid NLM and EMD []. (a) IR Fault-0.007in, (b) IR Fault-0.014in, (c) IR Fault-0.021in, (d) OR Fault0.007 in, (e) OR Fault-0.014in and (f) OR Fault-0.021in.

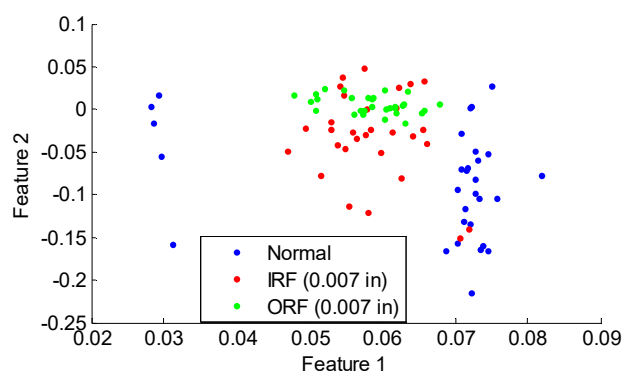

Fig. 6. Sample distribution of three example classes.

multimodal dimensional reduction methods, WKLFDA, PSOWKLFDA and I-PSO-WKLFDA (OAA-PSO-WKLFDA and OAO-PSO-WKLFDA), in which the I-PSO-WKLFDA algorithm is particularly concerned. To validate the effectiveness of the proposed feature extraction algorithms, we compared classification performance of the proposed method with seven other state-of-the-art feature analysis approaches, including global FDA, global TRLDA, global LFDA, global KLFDA (RBF kernel), global WKLFDA, global PSO-KLFDA (PSO is used to identify the sigma parameter of RBF kernel) and global PSO-WKLFDA algorithms. For LFDA, KLFDA

and WKLFDA, the nearest neighbor parameter $k(-$ th $)$ were chosen as $k(-$ th $)=7$.

To evaluate the performance of the methods, the extracted feature vectors are used as input for the SVM classifier to obtain classification accuracy. The classification accuracy in this study is computed as follows: 
$C_{\text {accuracy }}=\frac{\sum_{L} N_{T P}}{N_{\text {samples }}} \times 100(\%)$

where $L$ is the number of classes $(\mathrm{L}=10)$ in this study, $N_{T P}$ is the number of true positives (TP), defined as the total number of faults in class $i$ that are correctly classified as class $i$, and

$N_{\text {samples }}$ is the total number of samples used to evaluate the performance of the proposed bearing failure diagnosis.

To estimate the generalized classification accuracy, l-fold $\mathrm{CV}$, described in section II.D, is also employed in this validation step. In the $l$-fold $\mathrm{CV}$ estimation procedure, the training data is randomly divided into $l$ equal size folds. The classification performance is measured using one fold for

TABLE III

CONFUSION MATRIX FOR SHOWING CLASSIFICATION RESULTS OF THE PROPOSED OAO-PSO-WKLFDA

\begin{tabular}{|c|c|c|c|c|c|c|c|c|c|c|}
\hline & NM & ORF1 & IRF1 & BF1 & ORF2 & IRF2 & BF2 & ORF3 & IRF3 & BF3 \\
\hline NM & 2916 & 3 & 5 & 0 & 7 & 0 & 39 & 0 & 0 & 26 \\
\hline ORF1 & 15 & 2950 & 10 & 0 & 0 & 0 & 23 & 0 & 0 & 2 \\
\hline IRF1 & 0 & 0 & 2983 & 0 & 0 & 0 & 5 & 0 & 11 & 5 \\
\hline BF1 & 0 & 0 & 1 & 2985 & 0 & 0 & 0 & 13 & 0 & 0 \\
\hline ORF2 & 0 & 0 & 1 & 6 & 2989 & 0 & 4 & 0 & 0 & 0 \\
\hline IRF2 & 0 & 4 & 0 & 0 & 0 & 2996 & 0 & 0 & 0 & 5 \\
\hline BF2 & 18 & 0 & 0 & 0 & 4 & 4 & 2929 & 34 & 0 & 0 \\
\hline ORF3 & 24 & 23 & 0 & 2 & 0 & 0 & 0 & 2946 & 5 & 0 \\
\hline IRF3 & 2 & 1 & 0 & 7 & 0 & 0 & 0 & 7 & 2984 & 0 \\
\hline BF3 & 25 & 19 & 0 & 0 & 0 & 0 & 0 & 0 & 0 & 2962 \\
\hline Sensitivity(\%) & 97.20 & 98.33 & 99.43 & 99.5 & 99.63 & 99.87 & 97.63 & 98.20 & 99.47 & 98.73 \\
\hline Specificity(\%) & 99.70 & 99.80 & 99.92 & 99.95 & 99.96 & 99.97 & 99.98 & 99.80 & 99.94 & 99.84 \\
\hline
\end{tabular}

TABLE II

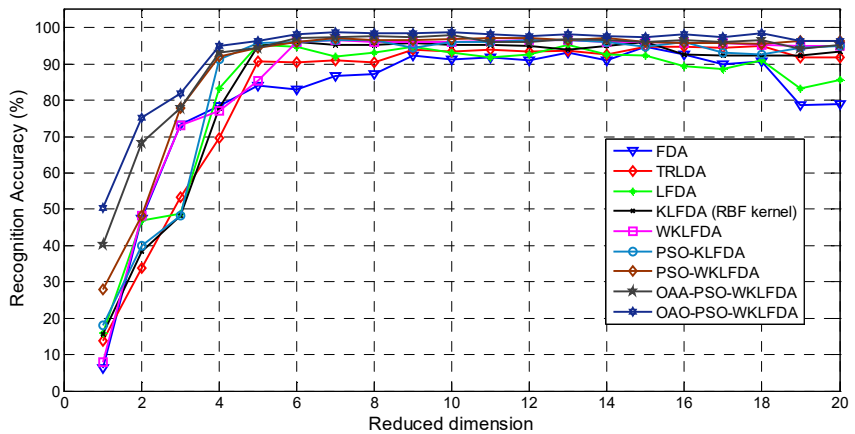

Fig. 7. Recognition accuracy versus reduced dimensionality using 25 training samples.

training, and the remaining folds for testing. In this experiment, $l$ is set to 4 . This is a reasonable compromise considering the computational complexity and modeling robustness. More specifically, 100 samples of created feature vectors are divided into four mutual folds (each fold includes 25 randomly samples for each bearing condition), and one fold (25 samples for each bearing condition) are used for the training set. The remaining folds $(75$ samples for each bearing condition) are used as the testing set. To obtain precise classification results, $l$-fold $\mathrm{CV}$ is performed ten times in this study. To select the WKLFDA parameters using PSO, the training set is used. In our experiments, the PSO had a population size set at 20 particles, with $c_{1}$ and $c_{2}$ both set to 2.0. The inertial weight decreased linearly from 0.9 to 0.4 over the number of iterations, as defined in (24). To select the parameters for each binary SVM classifier, grid search algorithm is employed. In this paper, the searching range of parameters are $C \in\left\{2^{-3}, 2^{-2}, 2^{-1}, 2,2^{2}, 2^{3}, 2^{6}, 2^{8}, 2^{10}, 2^{13}\right\}$ and $\gamma \in\left\{2^{-3}, 2^{-2}, 2^{-1}, 1,2,2^{2}, 2^{3}, 2^{4}, 2^{5}, 2^{6}\right\}$. Finally, the grid search algorithm gives the best combination of pair $\{C, \gamma\}$, yielding the highest classification accuracy.

Fig. 7 show the test accuracies of the dimensional reduction method in the $d$-dimensional subspace, where $d=1,2, \ldots, 20$. From Fig. 7 we can see that the performance of each method varies with reduced dimensions. The mean and best results of each method are also reported in Table II for easy in comparison. The subspaces according to the best records are
PERFORMANCE COMPARISON (\%) AMONG MULTIMODAL DIMENSIONAL REDUCTION METHODS

\begin{tabular}{|l|c|c|c|}
\hline \multirow{2}{*}{ Methods } & \multicolumn{3}{|c|}{ SVM } \\
\cline { 2 - 4 } & Mean & Max & Position \\
\hline FDA & 81.12 & 94.67 & 15 \\
\hline TRLDA & 82.78 & 95.00 & 18 \\
\hline LFDA & 83.02 & 95.33 & 13 \\
\hline KLFDA & 84.57 & 96.00 & 6 \\
\hline WKLFDA & 86.51 & 96.16 & 12 \\
\hline PSO-KLFDA & 86.20 & 96.70 & 8 \\
\hline PSO-WKLFDA & 89.34 & 97.20 & 7 \\
\hline $\begin{array}{l}\text { OAA-PSO- } \\
\text { WKLFDA }\end{array}$ & 91.07 & 97.80 & 10 \\
\hline $\begin{array}{c}\text { OAO-PSO- } \\
\text { WKLFDA }\end{array}$ & 93.38 & 98.80 & 8 \\
\hline \multicolumn{2}{|c|}{} & & \\
\hline
\end{tabular}

assigned as the optimal subspaces. Observing from Fig. 7 and Table II, we conclude that the I-PSO-WKLFDA (OAA-PSOWKLFDA and OAO-PSO-WKLFDA) method yields the highest accuracy, in which the OAO-PSO-WKLFDA provides better performance than OAA-PSO-WKLFDA. The experimental results coincided with theoretical analysis and can be explained as follows. Due to the limitation in the data, in which samples in a class are multimodal or samples between classes are nonlinearly separated in the input space, FDA generates the worst accuracy, $81.12 \%$. Using the trace ratio criteria instead of the ratio trace criteria to obtain transformation, the TRLDA $(82.78 \%)$ perform betters than 
FDA. By preserving the information within a class, the LFDA $(83.01 \%)$ provides better performance compared to FDA. By employing a kernel trick to map the data into higher dimension in which the nonlinear data possibly becomes linear separation, the KLFDA (84.57\%), WKLFDA (86.51\%), PSO-KLFDA (86.20\%) and PSO-WKLFDA (89.34\%) have much better performances. However, due to the capability of the wavelet kernel over the RBF kernel to approximate the nonlinear function, WKLFDA and PSO-WKLFDA yields better performance compared to KLFDA and PSO-KLFDA, respectively. Meanwhile, by using PSO to select the optimized WKLFDA parameters corresponding to the reduced dimension, the proposed PSO-WKLFDA algorithm yields better performance than WKLFDA. However, due to the capability of the wavelet kernel over the RBF kernel to approximate the nonlinear function, WKLFDA and PSO-

TABLE IV

PERFORMANCE COMPARISON (\%) BETWEEN FEATURE TRANSFORMATIONA AND FEATURE SELECTION METHODS

\begin{tabular}{|l|c|}
\hline \multicolumn{1}{|c|}{ Fault diagnosis methods } & $\begin{array}{c}\text { Classification } \\
\text { accuracy }(\%)\end{array}$ \\
\hline $\begin{array}{l}\text { Three selected features (kurtosis, crest } \\
\text { factor, skewness) of the raw vibration } \\
\text { signal }\end{array}$ & 76.50 \\
\hline Nine features of the raw vibration signal & 89.10 \\
\hline All 49 features & 82.83 \\
\hline DET-Feature selection & 94.10 \\
\hline OAO-DET-Feature selection & 95.56 \\
\hline PSO-FS & 96.40 \\
\hline OAO-PSO-FS & 98.17 \\
\hline PSO-WKLFDA & 97.20 \\
\hline OAO-PSO-WKLFDA & 98.80 \\
\hline
\end{tabular}

WKLFDA yields better performance compared to KLFDA and PSO-KLFDA, respectively. Meanwhile, by using PSO to select the optimized WKLFDA parameters corresponding to the reduced dimension, the proposed PSO-WKLFDA algorithm yields better performance than WKLFDA. However, the use of single global PSO-WKLFDA transformation does not give excellent accuracy due to the data overlap in the projected subspace. Using an individual approach, the data show less overlap in the projected subspace. Therefore, OAA-PSO-WKLFDA (91.07\%) and OAO-PSO-WKLFDA (93.38\%) yields higher accuracy and outperforms other methods. Since the OSO-PSO-WKLFDA provides the highest classification accuracy, it is suggested as an efficient method for bearing defect classification. Confusion matrix for showing classification results at the max value $(98.80 \%)$ of the proposed OAO-PSO-WKLFDA is described in Table III.

Finally, we compare the performance of the proposed feature transformation algorithm with the lower dimensional data and feature selection methods. In general, feature selection methods are classified into two categories: the filter and wrapper methods. In the filter methods, the optimal features are selected based on evaluation criteria, which does not require feedback from the classification system. In this paper, distance evaluation technique-based feature selection (DET-FS), which has been developed for bearing fault diagnosis [12], is used to compare. Wrapper methods use classification accuracy to evaluate feature subsets. Based on the wrapper method, the uses of intelligent optimization algorithms such as PSO have been proposed in conjunction with an intelligent classifier for feature selection, called PSObased feature selection (PSO-FS) [12]. In addition, to justify the approach transforming the vibration signals into high dimension data, we compare the proposed method with the approach which used lower dimensional data. The comparison results are shown in Table IV. From the results, we can see that the used of the three selected features (kurtosis, skewness and crest factor) as the input of SVM classifier, the classification accuracy is just $76.50 \%$. When using nine features extracted from the raw vibration signal, the classification accuracy is increased up to $89.10 \%$. This means that the set of nine features provides more information than that of the set of three features. Due to the extracted feature set of 45 features contains more irrelevant and redundant features compared to the used of nine features, the accuracy is just $82.83 \%$. This is an evaluation motivation for us to study the application of feature selection and dimensional reduction. When the DET-FS and PSO-FS are employed to select the feature subset, the classification accuracy is increased up to $94.10 \%$ and $96.40 \%$, respectively. Using our proposed dimensional reduction technique, PSO-WKLFDA, the classification accuracy is higher, $97.20 \%$. If we employ the individual feature selection technique for DET-FS and PSO$\mathrm{FS}$, yielding two new feature selection techniques, OAODET-FS and OAO-PSO-FS, the classification accuracy is increased up to $95.56 \%$ and $98.17 \%$, respectively. Meanwhile, the proposed method OAO-PSO-WKLFDA outperforms other method with classification accuracy up to $98.80 \%$.

\section{CONCLUSION}

This paper started by analyzing the performance of the conventional frequency domain analysis for different vibration signals. The results show that the technique fails to identify the BCF for some noisy vibration signals. Then, the distribution of the bearing data was analyzed. The results show that the distribution of the bearing data is a type of multimodal data. Thus, to enhance defect classification performance, a new multimodal dimensional reduction method, namely I-PSO-WKLFDA, was proposed. First, fault features based on NLM and EMD methods, was computed to represent diverse symptoms of bearing defects. The multiclass bearing defect classification problem was then converted to a multi-binary class bearing defect classification problem based on an OAO strategy. The I-PSO-WKLFDA was then applied for each binary class to extract the corresponding effective features. The effective features were used as the input to the SVM classifier. Finally, a decision fusion mechanism was employed to merge the classification results from each SVM classifier to identify the bearing condition. Experimental results indicated that the proposed fault diagnosis methodology achieves excellent classification accuracy. On 
the other hand, the main contribution of this paper is to propose a new effective dimensionality reduction method, thus the proposed methods can be applied for other pattern recognition problems.

\section{REFERENCES}

[1] M. Kang, J. Kim, J. M. Kim, "High-performance and energy efficient fault diagnosis using effective envelope analysis and de-noising on a general purpose graphics processing units," IEEE Trans. Pow. Electron. vol. 30, no. 5, pp. 2763-2776, 2015.

[2] W. Li, S. Zhang, S. Rakheja, "Feature denoising and nearest-farthest distance preserving projection for machine fault diagnosis," IEEE Trans. Ind. Inform., doi: 10.1109/TII.2015.2475219, 2015.

[3] S. Lu, Q. He, F. Hu, F. Kong, "Sequential multiscale noise tuning stochastic resonance for train bearing fault diagnosis in an embedded system," IEEE Trans. Inst. Meas., vol. 63, no. 1, pp. 106-116, 2014.

[4] M.D.Prieto, G.Cirrincione, A.G.Espinosa, J.A.Ortega, Henao, H., "Bearing Fault Detection by a Novel Condition-Monitoring Scheme Based on Statistical-Time Features and Neural Networks," IEEE Trans. Ind. Electron., vol.60, no.8, pp.3398-3407, Aug. 2013.

[5] M. Amar, I. Gondal, C. Wilson, "Vibration spectrum imaging: A novel bearing fault classification approach," IEEE Trans. Ind. Electron. vol.62, no.1, pp.3398-3407, Aug. 2013.

[6] H. Zoubek, S. Villwock, and M. Pacas, "Frequency response analysis for rolling-bearing damage diagnosis," IEEE Trans. Ind. Electron., vol. 55, no. 12 , pp. 4270-4276, Dec 2008.

[7] M. Kang, J. Kim, L. M. Wills, J. M. Kim, "Time-varying and multiresolution envelop analysis and discriminant feature analysis for bearing fault diagnosis," IEEE Trans. Indus. Electron., doi 10.1109/TIE.2015.2460242, 2015.

[8] Y. Lei, J. Lin, Z. He, M. J. Zou, "A review on empirical mode decomposition in fault diagnosis of rotating machinery", Mech. Syst. Singal Process., vol. 35, no. 1-2, pp. 108-126, 2013.

[9] M. Van, H. J. Kang and K. S. Shin, "Rolling element bearing fault diagnosis based on nonlocal means de-noising and empirical mode decomposition", IET Sci. Meas. and Technol., vol. 8, no. 6, 2014.

[10] T. W. Rauber, F. de Assis Boldt, F. M. Varejao, "Heterogeneous feature selection models and feature selection applied to bearing fault diagnosis," IEEE Trans. Ind. Electron., vol. 62, no. 1. pp. 637-646, 2015.

[11] M. Kang, J. Kim, J. -M. Kim, A. C. C. Tan, E. Y. Kim, and B. -K. Choi, "Reliable fault diagnosis for low-speed bearings using individual trained support vector machines with kernel discriminative feature analysis," IEEE Trans. Power Electr., vol. 30, no. 5, pp. 2786-2797, 2015

[12] M. Van, H. J. Kang, "Bearing fault diagnosis using non-local means algorithm and empirical mode decomposition-based feature extraction and two-stage feature selection," IET Sci. Meas. and Technol., DOI: 10.1049/iet-smt.2014.0228, 2015.

[13] A. M. Martinez and A. A. Kak, "PCA versus LDA," IEEE Trans. Pattern Anal. Mach. Intell, vol. 23, no. 2, pp. 228-233, Feb. 2001.

[14] X. Jin, M. Zhao, Tommy W. S. Chow, and M. Pecht, "Motor bearing fault diagnosis using trace ratio linear discriminant analysis," IEEE Trans. Ind. Electron., vol. 61, no. 5, pp. 2441-2451, May. 2014.

[15] C. Li, R. V. Sanchez, G. Zurita, M. Cerrada, D. Cabrera, "Multimodal deep support vector classification with homologous features and its application to gearbox fault diagnosis," Neurocomputing, vol. 168, pp. 119-127, 2015.

[16] M. Sugiyama, "Dimensionality reduction of multimodal labeled data by local fisher discriminant analysis," J. Mach. Learn. Res., vol. 8, pp. 1027-1061, May. 2007.

[17] W. Li, S. Prasad, J. E. Fowler, and L. M. Bruce, "Decision fusion in kernel-induced spaces for hyperspectral image classification," IEEE Trans. Geosci. Remote. Sens., vol. 52, no. 6, pp. 3399-3411, Jun. 2014

[18] M. Van, H. J. Kang, "Wavelet kernel fisher discriminant analysis with particle swarm optimization algorithm for bearing defect classification," IEEE Trans. Int. Mea., doi: 10.1109/TIM.2015.2450352, 2015.

[19] Q. H. Zhan and A. Benveniste, "Wavelet networks," IEEE Trans. Neural. Netw., vol. 3, no. 6, pp. 889-898, Nov. 1992.
[20] M. Shen, L. Lin, J. Chen, C. Q. Chang, "A prediction approach for multichannel EEG signals modeling using local wavelet SVM," IEEE Trans. Inst. Meas., vol.59, no.5, pp.1485-1492, May. 2010.

[21] J. Kennedy and R. C. Eberhart, "Particle Swarm optimization," in Proc. IEEE Int. Conf. Neural Netw., Perth, Australia, 1995, pp. 1942-1948.

[22] C. W. Hsu and C. J. Lin, "A comparison of methods for multiclass support vector machines," IEEE Trans. Neural Networ., vol. 13, no. 2, pp. 415-425, 2011.

[23] L. Zelnik-Manor and P. Perona, "Self-tuning spectral clustering" Proc. $18^{\text {th }}$ Ann. Conf. Advances in Neural Information Processing Systems 17, pp.1601-1608, 2004.

[24] J. D. Rodriguez, A. Perez, J. A. Lozano, "Sensitivity analysis of k-fold cross validation in prediction error estimation" IEEE Trans. Pattern Anal. Mach. Intell., vol. 29, no. 4, pp.650-664, 2010.

[25] K. A. Loparo: 'Bearing data center', http://www.eecs.case.edu/laboratory/bearing, Case Western Reserve University, accessed 2014.

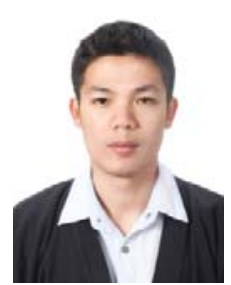

Mien Van received his B.S. degree in Electrical Engineering from Danang University of Technology, DaNang city, Vietnam, in 2009. He received his Ph.D. degree in the School of Electrical Engineering, University of Ulsan, Korea in 2015.

He is currently a Post-Doctoral Research Fellow with the Advanced Robotic Centre, Faculty Engineering, National University of Singapore, Singapore. His research interests include automatic control, fault diagnosis and fault tolerant, machine learning, robot-vision system and sensing and perception.

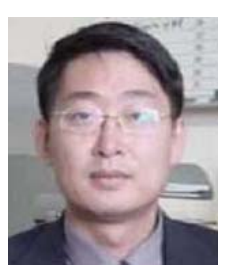

Hee-Jun Kang received his B.S. degree in Mechanical Engineering from Seoul National University, Korea in 1985. He received his M.S and Ph.D. degrees in Mechanical Engineering from The University of Texas at Austin, USA in 1988 and 1991, respectively. Since March in 1992, he has been a Professor of Electrical Engineering in University of Ulsan. His current research interests are sensor based robotic application, robot calibration, haptics, robot fault diagnosis and mechanism analysis. 